\title{
Radioimmunoassay of Human Apolipoprotein CII
}

\author{
A STUDY IN NORMAL AND HYPERTRIGLYCERIDEMIC SUBJECTS
}

\author{
M. L. Kashyap, L. S. Srivastava, C. Y. Chen, G. Perisutti, M. Campbell, \\ R. F. LuTMER, and C. J. GLuECK
}

From the Lipid Research Clinic, The Radioimmunoassay Laboratory of the Division of Metabolism, and General Clinical Research Center, Department of Medicine, University of Cincinnati, College of Medicine, Cincinnati, Ohio 45267

A B S TRACT A specific, precise, and sensitive double-antibody radioimmunoassay for the measurement of human apolipoprotein CII (apoCII) was developed. ApoCII was labeled with ${ }^{125}$ I (chloramineT) and monospecific antibody was raised in rabbits. No appreciable cross-reactivity with apolipoproteins CI, CIII, AI, AII, low density lipoproteins, and lipoprotein-free plasma was observed. Lipoporteins containing apoCII displaced the standard curve in parallel. ApoCII measurement was not affected by pretreatment of plasma with tetramethylurea, ethanol-diethyl ether, or heating.

Mean $( \pm$ SE) plasma-immunoreactive apoCII in 47 normotriglyceridemic subjects was $51.8 \pm 3.2 \mu \mathrm{g} / \mathrm{ml}$, generally comparable with previous estimates of its concentration by other methods. ApoCII levels in 9 subjects with type IIB lipoprotein pattern, 14 with the type IV lipoprotein pattern, and 5 with type V

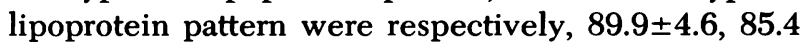
$\pm 6.9,132.8 \pm 21.0 \mu \mathrm{g} / \mathrm{ml}$, all higher than normals ( $P$ $<0.001)$. Plasma apoCII and triglyceride concentrations correlated in normo- and hypertriglyceridemics ( $r=0.36$ and 0.58, $P<0.05$ ). Plasma triglycerides correlated inversely with the fraction of total apoCII in very low density lipoprotein (VLDL)-free plasma $(r=-0.75, P<0.01)$. There was no correlation between plasma apoCII and high density lipoprotein cholesterol.

In normotriglyceridemics, VLDL apoCII levels correlated with in vitro lipoprotein lipase (LPL) activa-

Received for publication 31 December 1976 and in revised form 16 March 1977.

Part of this work was presented at the Annual Meeting of the Midwest Section of the American Federation for Clinical Research at Chicago, Ill., 4 November 1976 (1).

A portion of this work was done during Dr. Glueck's tenure as Established Investigator of the American Heart Association, 1971- 1976. tor activities $(r=0.89, P<0.01)$. In hypertriglyceridemic subjects the mean concentrations of apoCII per milligrams VLDL protein, LPL activator activity per milligrams VLDL protein, and LPL activator activity per micrograms VLDL apoCII were all lower than in normotriglyceridemics, $P<0.05$.

As plasma triglycerides and apoCII increase, apoCII is redistributed from high density lipoprotein to VLDL. However, the amount of apoCII per milligram VLDL protein and its LPL activator potency per milligram VLDL protein are reduced. These factors may contribute to impaired VLDL catabolism.

\section{INTRODUCTION}

Plasma triglycerides in humans are principally transported by chylomicrons after fat ingestion, and by very low density lipoproteins (VLDL) ${ }^{1}$ during fasting. Triglyceride catabolism is mediated by lipoprotein lipases (LPL) which hydrolyze chylomicron and VLDL triglyceride into glycerol and fatty acids. LPL are activated and probably inhibited by a group of apolipoproteins (2-4). Apolipoprotein CII (apoCII) is generally accepted as the major activator of LPL from various sources: human adipose tissue (4), the human heart (5), human and rat post-heparin plasma LPL $(6,7)$, bovine milk LPL $(6,8)$, and rat adipose tissue (7). An apoCI-activated LPL from post-heparin plasma has been described (9). Conversely, certain apolipoproteins in VLDL and high density lipoproteins (HDL): CI, CIII, AI, and E inhibit human adipose tissue LPL (4).

\footnotetext{
${ }^{1}$ Abbreviations used in this paper: apoA, B, C, and E, apolipoproteins A, B, C, E: HDL, high density lipoproteins; ILDL, intermediate low density lipoproteins; LDL, low density lipoproteins; LPL, lipoprotein lipase; TMU, tetramethylurea; VLDL, very low density lipoproteins.
} 
The $\mathrm{C}$ apolipoproteins are found in HDL and VLDL. After fat ingestion in humans, apoCII and LPL activator property are transferred from HDL to chylomicrons, with HDL functioning as an apoCII reservoir, contributing activator to potentiate triglyceride hydrolysis (10). As chylomicrons and VLDL are catabolized, $\mathrm{C}$ apolipoproteins are lost, presumably to HDL, and low density lipoprotein (LDL) is formed $(11,12)$. If apoCII is deficient in plasma (cannot be transferred from HDL to VLDL or suboptimally activates LPL) hypertriglyceridemia may result. Breckenridge et al. (13) have recently described a severely hypertriglyceridemic patient with absent apoCII (polyacrylamide gel electrophoresis) whose plasma failed to activate LPL in vitro. Thus, the $\mathrm{C}$ apolipoproteins play a very important role in triglyceride metabolism.

To enhance understanding of the possible role of apoCII in the pathophysiology of the hypertriglyceridemias, a sensitive, accurate, precise, and specific method for its measurement is necessary. Miller and Schonfeld (14) have reported the development of a radioimmunoassay for apoCII measurement. In this preliminary report they found that the content of apoCII in VLDL and HDL protein was 10-13 and $1-2 \%$, respectively. The other methods for apoCII quantitation currently available have utility but also disadvantages. Havel and associates have used in vitro systems with various LPL sources and quantitation of the LPL activator property of serum, plasma, or various subfractions $(2,10)$. Subsequently, other quantitative bioassays based on the activator property have been described $(15,16)$. They indicate net LPL activator concentration of a given sample, and are not specific for apoCII.

Lipoprotein apoCII content has been quantitated by Kane et al. (17) using tetramethyl urea (TMU) for delipidation and separation of constituent polypeptides by polyacrylamide gel electrophoresis. This method does not allow measurement of the total plasma apoCII and may have limitations in its sensitivity.

Electroimmunoassays or radial immunodiffusion methods are generally less sensitive than the radioimmunoassay and require relatively large volumes of antibody. These considerations are important in apoCII measurement since this apolipoprotein may have the lowest concentration among human plasma apolipoproteins (18) and is biologically active in very small concentrations.

This paper describes a sensitive, specific, precise, and accurate radioimmunoassay for quantitation of apoCII in plasma and in lipoprotein subfractions, and provides comparison with the LPL activator property of the same plasmas and lipoprotein subfractions. The utility of the radioimmunoassay as a tool for assessment of hypertriglyceridemia is also evaluated.

\section{METHODS}

\section{The radioimmunoassay}

Antigen preparation. Blood was collected in EDTA tubes $(1 \mathrm{mg} / \mathrm{ml})$ from heatlhy fasting $(12-14 \mathrm{~h})$ normal volunteers who were on no medication; plasma was separated in a refrigerated centrifuge. VLDL was isolated by preparative ultracentrifugation according to Havel's method (19) using a 40.3 rotor and a L5-50 Beckman ultracentrifuge (Beckman Instruments, Inc., Spinco Div., Palo Alto, Calif.). VLDL from the first ultracentrifugal run was layered with normal saline and respun a second time. ApoCII was isolated from this VLDL according to the method of Brown et al. (20) by sequential dialysis, lyophilization, delipidation with ethanol-diethyl ether, solubilization of the apoVLDL in tris buffer containing sodium dodecyl sulfate, Sephadex gel chromatography, and DEAE ion-exchange chromatography. The second peak from this step was dialyzed against $0.05 \mathrm{M}$ ammonium bicarbonate and lyophilized. The amino acid composition of this fraction was determined using the Durrum D-500 amino acid analyzer (Durrum Instrument Corp., Sunnyvale, Calif.) with results similar to those obtained by Brown et al. (21). Polyacrylamide gel electrophoresis in urea (17) and sodium dodecyl sulfate (22) revealed a single band.

Antibody preparation. Female albino New Zealand rabbits weighing approximately $2.5 \mathrm{~kg}$ were immunized with apoCII emulsified with complete Freund's adjuvant. Injections were given directly into the popliteal lymph nodes (23) and also intradermally in multiple sites on the back (24). Booster injections on the back were given twice, at approximately 3 wk apart, and then the animals were bled 14 days after the last injection. The serum was kept at $-20^{\circ} \mathrm{C}$.

Iodination of apoCII with ${ }^{125}$. Basically the method of Greenwood et al. (25) was used. To $7 \mu \mathrm{g}$ of lyophilized apoCII, $0.1 \mathrm{ml}$ of $0.5 \mathrm{M}$ phosphate buffer, $\mathrm{pH} 7.6$ was added and the tube gently tapped to dissolve the apoprotein. This was followed by the addition of $0.5 \mathrm{mCi}{ }^{125} \mathrm{I}$ and 87.5 $\mu \mathrm{g}$ of chloramine-T in $25 \mu \mathrm{l}$ of $0.05 \mathrm{M}$ phosphate buffer, $\mathrm{pH}$ 7.6. After $30 \mathrm{~s}, 250 \mu \mathrm{g}$ of sodium metabisulfite in 0.1 $\mathrm{ml}$ of $0.05 \mathrm{M}$ phosphate buffer, $\mathrm{pH} 7.6$ was added to stop the reaction. $50 \mu \mathrm{l}$ of $7 \%$ bovine serum albumin was then added. The reactant mixture was then subjected to gel chromatography using Sephadex G-100 (a $1 \times 100$-cm column previously coated with $3 \%$ bovine serum albumin) and equilibrated with $0.07 \mathrm{M}$ barbital buffer at pH 8.6. The elution profile showed three major peaks as shown in Fig. 1. The following procedures were done to assess homogeneity of the labeled apoCII. When mixed with unlabeled apoCII both eluted in the same peak on gel chromatography, were noted as a single band on polyacrylamide gel electrophoresis. Thin gel slices subjected to scintillation counting also revealed a single peak which corresponded to the band stained with Coomassie Blue. The percent binding of labeled protein from the first and second peaks with antibody diluted $1: 2,000$ was 14 and $79 \%$, respectively. With an antibody dilution of 1:10,000, the percent binding of labeled protein from the second peak was $48 \%$. The specific activity of the labeled apoCII ranged between 87 and $107 \mathrm{mCi} / \mathrm{mg}$.

The assay. The assay was done with a $10 \times 75-\mathrm{mm}$ Kimble (Kimble Products Div., Owens-Illinois, Inc., Toledo, Ohio) tube. To $0.4 \mathrm{ml}$ of $1 \%$ bovine serum albumin in $0.02 \mathrm{M}$ barbital buffer ( $\mathrm{pH} 8.5$ containing $1 \%$ sodium azide), the following were added in order: (a) $0.1 \mathrm{ml}$ plasma or unknown sample diluted 1:50-1:400 with barbital buffer or standard unlabeled apoCII (for the standard curve); $(b)$ 


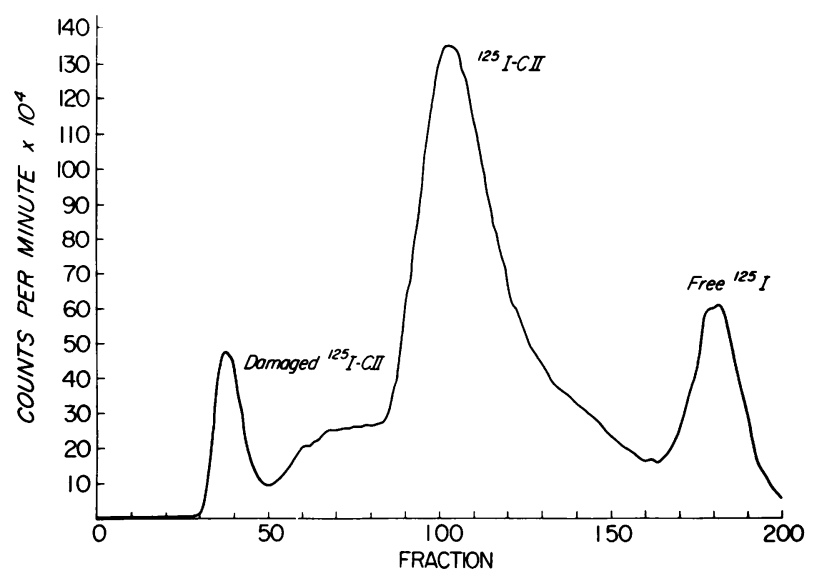

FIGURE 1 Elution profile of apoCII after labeling with ${ }^{125}$ I and separation of products in Sephadex G-100 column. The second peak was used as tracer in the radioimmunoassay system.

$0.1 \mathrm{ml}$ antibody diluted 1:10,000; (c) $0.1 \mathrm{ml}$ of ${ }^{125} \mathrm{I}$-apoCII containing approximately $10,000 \mathrm{cpm}$.

The assay was done with tubes in ice throughout. The mixture was incubated for $72 \mathrm{~h}$ at $4^{\circ} \mathrm{C}$ and then $0.1 \mathrm{ml}$ of sheep anti-rabbit gamma globulin (diluted to give maximum precipitation) was added to each tube. Further incubation for $48 \mathrm{~h}$ at $4^{\circ} \mathrm{C}$ was followed by centrifugation in a refrigerated centrifuge at $2,500 \mathrm{rpm}$ for $20 \mathrm{~min}$. The supernate was aspirated and counts from the precipitate were measured in a Packard Autogamma scintillation spectrometer $\mathbf{5 2 3 0}$ (Packard Instrument Co., Inc., Downers Grove, Ill.).

\section{The LPL activator assay}

The in vitro LPL activator property of plasma and its subfractions was measured by an adaptation of the methods of Havel et al. (2) and Schotz et al. (26). The method was similar to that of Rogers et al. (16) and Chu et al. (15) except that milk LPL and sonicated triolein were used. The detailed procedure used in this study was as follows:

A mixture of $4.0 \mu \mathrm{Ci}$ of $\left[{ }^{14} \mathrm{C}\right]$ triolein and $0.108 \mathrm{~g}$ of $\left[{ }^{12} \mathrm{C}\right]$ triolein was placed in a $50-\mathrm{ml}$ beaker. This amount of triolein provided excess unlabeled substrate throughout the period of hydrolysis, not affected by variation in the amount of added VLDL triglycerides. The mixture was dried down under a stream of nitrogen. To this $3.6 \mathrm{ml}$ of $1 \%$ bovine serum albumin, $3.6 \mathrm{ml}$ of $1 \%$ Triton $\mathrm{X}-100$ and $10.8 \mathrm{ml}$ of $0.2 \mathrm{M}$ Tris-HCl buffer, pH 8.0 were added. The beaker was placed in a bucket of ice. Immediately before substrate preparation, the mixture was sonicated for exactly 4 min (in two 2-min bursts) at no. 5 on the Branson model W185D sonifier cell disruptor (Branson Sonic Power Co., Danbury, Conn.).

The enzyme extract was made by adding $\mathrm{NH}_{4} \mathrm{OH}$ to $0.4 \mathrm{~g}$ of milk LPL prepared according to Bier and Havel (27) with a final volume of $10 \mathrm{ml}$. The mixture was mixed gently for 3 min with a vortex shaker. It was then centrifuged for $10 \mathrm{~min}$ at $3,000 \mathrm{rpm}\left(\right.$ at $4^{\circ} \mathrm{C}$ ). The supernate was saved for the enzyme in the assay system.

For a single assay the following were added: $0.3 \mathrm{ml}$ of serum cofactor standard, blank ( $0.9 \%$ sodium chloride) or unknown sample, $0.9 \mathrm{ml}$ of $0.9 \%$ sodium chloride, $0.6 \mathrm{ml}$ of sonicated substrate, and $0.2 \mathrm{ml}$ of enzyme preparation. The volume of $0.9 \%$ sodium chloride was increased if the sample volume was less than $0.3 \mathrm{ml}$ and decreased for a larger sample. A 15- or 18-ml test tube with a ground glassstoppered closure was used. After vortex mixing the tubes were then placed in a shaker bath at $37^{\circ} \mathrm{C}$ for $20 \mathrm{~min}$. The reaction was terminated by addition (and mixing) of $4 \mathrm{ml}$ of an isopropanol, $3 \mathrm{~N} \mathrm{H}_{2} \mathrm{SO}_{4} 40: 1$ solution. Lipids and fatty acids were extracted by adding $2 \mathrm{ml}$ distilled $\mathrm{H}_{2} \mathrm{O}$ and $5 \mathrm{ml}$ hexane. The tube was shaken end-to-end on a mechanical agitator for $2 \mathrm{~min}$. The extraction mixture was left to stand for $20 \mathrm{~min}$. A 5.0-ml aliquot of the upper (hexane) phase was added to $1 \mathrm{ml}$ of $0.1 \mathrm{~N} \mathrm{KOH}$ in a $15-\mathrm{ml}$ conical calibrated centrifuge tube with a ground glass-stoppered closure. The tube was then mixed for $10 \mathrm{~min}$ on an end-to-end shaker to extract the free fatty acids into the alkali.

The volume of the lower phase was recorded. $1 \mathrm{ml}$ of the lower (KOH) phase was dissolved in $5 \mathrm{ml}$ of Packard Instagel (Packard Instrument Co., Inc.,) and the radioactivity assayed in a Packard liquid scintillation counter. $0.6 \mathrm{ml}$ of the sonicated substrate was dissolved in $5 \mathrm{ml}$ of Instagel and used as the measurement of unhydrolyzed triolein standard.

The total radioactivity of the hexane extract minus the radioactivity from a blank containing saline was used to calculate the amount of triolein hydrolyzed in $20 \mathrm{~min}$. The percent $(\bar{x} \pm \mathrm{SE})$ of radioactivity in the blank to that given by 0.3 and $0.5 \mathrm{ml}$ of the standard plasma was $8.5 \pm 0.3$ and $4.7 \pm 0.2 \%$, respectively $(n=10)$. Preincubation of $L P L$ at $60^{\circ} \mathrm{C}$ for $30 \mathrm{~min}$ inactivated it. Incubation of plasma at $60^{\circ} \mathrm{C}$ did not affect cofactor activity. Addition of heat-inactivated enzyme to LPL, substrate, and serum did not alter the amount of triolein hydrolyzed indicating that there was probably no activator or inhibitor in the milk LPL preparation. The result can be expressed as micromoles of free fatty acids (FFA) per milliliters of medium per $20 \mathrm{~min}$ by assuming the liberation of $3 \mathrm{~mol}$ FFA $/ \mathrm{mol}$ triolein or it can be expressed as the amount of triolein hydrolyzed. We have chosen to express the results in the first method. All samples were run in duplicate.

Approximately $600 \mathrm{ml}$ of pooled serum was collected, divided into 1- and 2- $\mathrm{ml}$ aliquots and then frozen. A fresh tube of this serum was thawed for each experiment. A typical standard curve showing the relationship of the addition of increasing volumes of serum on activation of LPL in the above system is shown in Fig. 2. A similar curve was obtained with unknown samples of hyperlipemic

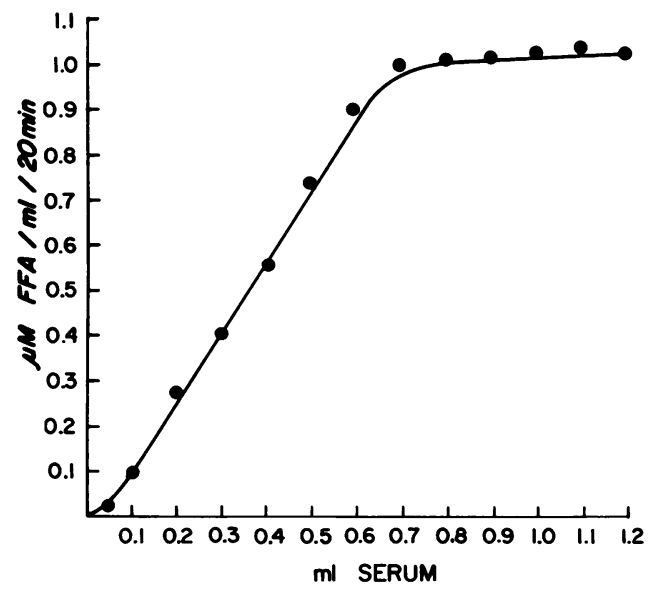

FIGURE 2 Effects of increasing volume of serum on liberation of free fatty acids from emulsified triolein in the presence of milk LPL. 


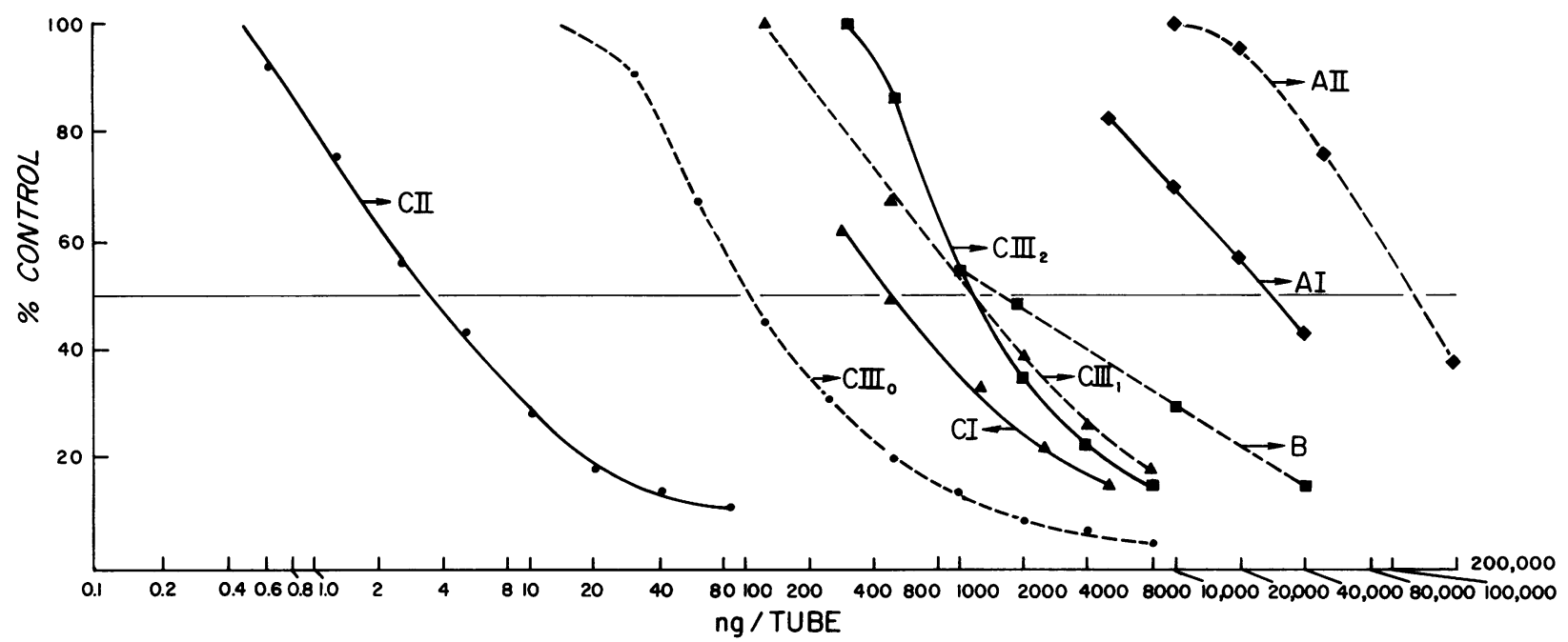

FIGURE 3 Displacement of tracer ( ${ }^{125} \mathrm{I}$-apoCII) by unlabeled apoCII, apoCI, apoCIII ${ }_{0}, \mathrm{CIII}_{1}$, $\mathrm{CIII}_{2}, \mathrm{AI}, \mathrm{AII}$, and apoB as low density lipoprotein (B).

plasma or VLDL. For each run, $0.1,0.3$, and $0.5 \mathrm{ml}$ of serum which corresponded to the straight upslope of the activation curve were included. The regression line for the three points was plotted from the regression equation. The pooled plasma was given an arbitrary value of $100 \mathrm{U} / \mathrm{ml}$. The concentration (in units per milliliter) of the unknown sample was read from this standard. An increased volume of the sample or its dilution with the $0.9 \%$ sodium chloride as necessary was performed to yield a value that was clearly within linear range of the standard. Serial sample dilution did not affect activator concentration, provided sample dilutions were within the range of linear portion of the activation curve. With fixed amounts of enzyme and the usual amount of labeled and unlabeled triolein, additional unlabeled triolein (within the range of the mass of triglyceride that was added in an unknown sample) was added. This did not decrease the amount of labeled fatty acids liberated in the system. There was no significant difference between plasma (EDTA used as anticoagulant) and serum.

The inter- and intra-assay coefficients of variation of this method were 3.9 and $2.9 \%$, respectively. The sensitivity of this method was $8 \mathrm{U} / \mathrm{ml}$ with the above-mentioned precision.

\section{Other analytical methods}

Proteins were determined according to Lowry's method (28). Cholesterol, triglycerides, lipoprotein electrophoresis, and plasma HDL cholesterol were measured with Autoanalyzer according to the Lipid Research Clinics Methodology (29). Preparative ultracentrifugation for isolating lipoproteins was performed according to Havel et al. (19). Except for VLDL, isolation of intermediate LDL (ILDL) $(d>1.006<1.019)$, LDL $(d>1.019<1.063), \mathrm{HDL}_{2}(d>1.063<1.125), \mathrm{HDL}_{3}$ $(d>1.125<1.210$, VHDL $(d>1.210<1.25)$, and lipoprotein-free plasma (residue) $(d>1.25)$ was followed by dialysis in $0.15 \mathrm{M}$ sodium chloride in $1 \mathrm{mM}$ disodium EDTA.

Organic solvent extraction of plasma and lipoproteins was performed according to Schonfeld et al. (30). TMU delipidates and solubilizes apolipoproteins except apoB $(10,31)$. We assessed the effect of TMU pretreatment on the apoCII content of plasma, and lipoproteins. TMU purchased from Burdick \& Jackson Laboratories Inc. (Muskegon, Mich., lot 49442) was freshly distilled in glass and added to an equal volume of plasma. When undiluted TMU was added, a dense semi-solid mass formed which did not permit accurate pipetting for subsequent dilutions. Subsequently, $4.2,1.0$, and $0.5 \mathrm{M}$ concentrations were used. The mixture was incubated at room temperature $\left(26^{\circ} \mathrm{C}\right.$ in a water bath) for $30 \mathrm{~min}$. In one experiment, the incubation was performed at $37^{\circ}$ and $52^{\circ} \mathrm{C}$ for $30 \mathrm{~min}$. The mixture was centrifuged at 2,500 rpm for $20 \mathrm{~min}$ and the apoCII concentration in the supernate was measured.

\section{Study subjects}

Studies were made in 32 normal healthy volunteers, and in 43 patients with primary and familial hyperlipoproteinemias who were classified according to Lipid Research Clinics criteria (32). There were no patients with familial types I or III hyperlipoproteinemia in this study. All patients fasted except for water for 12-14 h. Venous blood was drawn in tubes containing EDTA to give a blood concentration of $1 \mathrm{mg} / \mathrm{ml}$. After centrifugation, the plasma was refrigerated and the determination of plasma lipids, electrophoresis, and preparative ultracentrifugation was begun as soon as possible.

\section{Statistical methods}

Statistical significance of mean differences and correlation coefficients were calculated according to Snedecor and Cochran (33).

\section{RESULTS}

\section{ApoCII -radioimmunoassay}

Characterization of the apoCII antisera. Fig. 3 shows the displacement of the tracer by pure apoCII and other apolipoproteins. The counts per minute of a precipitate $(\cong 5,000 \mathrm{cpm})$ from a tube containing labeled apoCII and no unlabeled apoCII minus counts per minute precipitated in a nonspecific binding tubes 
was given a value $100 \%$ and regarded as control. The precipitate in nonspecific binding tubes contained $<3 \%$ of the label (range $=0.96-3.00 \%$ ). Crossreactivity with other apolipoproteins was defined as $x / y \times 100$ where $x=$ the amount of unlabeled apoCII required to displace $50 \%$ of the control; $y=$ the amount of another apolipoprotein required to also displace $50 \%$ of control. Apolipoproteins $\mathrm{CI}, \mathrm{CIII}_{0}, \mathrm{CIII}_{1}$, and $\mathrm{CIII}_{2}$ were obtained from VLDL during isolation of apoCII. Their identity was confirmed by amino acid analysis which showed a composition similar to that reported by Brown et al. (21). LDL was used to study cross-reactivity as apolipoprotein $\mathrm{B}$ is insoluble in the buffer system employed. The LDL was isolated as the density fraction between 1.024 and 1.050 from a normal healthy individual. Apolipoproteins AI and AII were isolated from delipidated purified HDL (34).

The percent cross-reaction of different apolipoproteins and LDL with apoCII antiserum was as follows: AI, $0.013 \%$; AII, $0.002 \%$; B (as LDL), $0.219 \%$; CI, 0.70\%; $\mathrm{CIII}_{0}, 3.182 \%$; $\mathrm{CIII}_{1}, 0.292 \%$; $\mathrm{CIII}_{2}, 0.292 \%$. All apolipoproteins and LDL showed a cross-reactivity of $<1 \%$ except apolipoprotein CIII $_{0}$ which showed a cross-reactivity of $3.18 \%$. We believe this to be secondary to a contamination of apolipoprotein $\mathrm{CIII}_{0}$ with apoCII which has been noted as a problem in the purification of apoCIII ${ }_{0}$ (35). Furthermore, the amino acid sequence of the CIII subspecies is the same (36). ApoCII was undetectable in lipoprotein-free plasma obtained from the infranate after a single ultracentrifugation of plasma at $d=1.250$.

VLDL, ILDL, $\mathrm{HDL}_{2}, \mathrm{HDL}_{3}$, and VHDL fractions showed displacement curves that were parallel to the standard curve using unlabled apoCII. The content of apoCII (as percent of total lipoprotein protein) in each of these fractions (from a normal subject) is shown in Table I.

Characteristics of the assay system. The system was sensitive enough to detect displacement with 0.6 ng of unlabeled apoCII in each tube. For the standard curve the working range was $1.56-50 \mathrm{mg}$. The mean coefficient of variation of seven standard curves was $4.8 \%$. Serial dilution of a given sample gave correspondingly lower values of the concentration of apoCII indicating that the apoCII was completely soluble and reactive in the undiluted sample. The inter- and intra-assay coefficient of variation of a given sample were 4.9 and $3.5 \%$, respectively.

Effect of preheating plasma on apoCII concentration. Karlin et al. (37) have shown that the concentration of radioimmunoassayable human apoAI is increased by heating plasma before measurement and found that the maximum increment occurs in plasma preheated for $3 \mathrm{~h}$ at $52^{\circ} \mathrm{C}$. Two plasma samples were incubated at $26^{\circ} \mathrm{C}$, and $52^{\circ} \mathrm{C}$ for $3 \mathrm{~h}$ in a constant
TABLE I

ApoCII Content of Total Protein of Lipoprotein Fractions of Human Plasma

\begin{tabular}{ccc}
\hline Lipoprotein fraction & Density & Content* \\
\hline & & $\%$ \\
VLDL & $<1.006$ & 12.30 \\
ILDL & $1.006-1.019$ & 0.81 \\
LDL & $1.019-1.063$ & 0.32 \\
HDL $_{2}$ & $1.063-1.125$ & 1.61 \\
HDL $_{3}$ & $1.125-1.210$ & 0.55 \\
VHDL & $1.210-1.250$ & 0.12 \\
\hline
\end{tabular}

* Expressed as percent of total protein in each density (d) fraction.

temperature bath. In sample 1 , apoCII concentration was 63.9 and $60.3 \mu \mathrm{g} / \mathrm{ml}$ at $26^{\circ}$ and $52^{\circ} \mathrm{C}$, in sample $2,62.4$ and $60.6 \mu \mathrm{g} / \mathrm{ml}$. Thus, heating had no effect on the concentration of total plasma apoCII. Aliquots of a third plasma sample were heated for $30 \mathrm{~min}$ at $26^{\circ}$, $37^{\circ}$, and $52^{\circ} \mathrm{C}$ with no appreciable change in apoCII concentration $(41.1,41.1,39.6 \mu \mathrm{g} / \mathrm{ml}$, respectively).

Effect of organic solvent extraction before apoCII measurement. Schonfeld et al. (30) found that the concentration of apoAI increased after pretreatment of plasma with ethanol-diethyl ether and solubilization of the protein precipitate in $8 \mathrm{M}$ urea. Two experiments were carried out to assess the effect of organic solvent extraction on apoCII measurement. Untreated and pretreated apoCII concentrations in two aliquots of a plasma sample were 47 and $36 \mu \mathrm{g} / \mathrm{ml}$. A purified VLDL sample containing $75 \mu \mathrm{g} / \mathrm{ml}$ apoCII measured $66 \mu \mathrm{g} / \mathrm{ml}$ after organic solvent extraction.

Effect of pretreatment of plasma with TMU on measurement of apoCII. No significant change in total plasma apoCII was noted after pretreatment of plasma with $4.2,1.0$, and $0.5 \mathrm{M} \mathrm{TMU}$ at $26^{\circ} \mathrm{C}$ for $30 \mathrm{~min}$. In three aliquots of a plasma sample after a 30 -min incubation with $4.2 \mathrm{M}$ TMU at $26^{\circ}, 37^{\circ}$, and $52^{\circ} \mathrm{C}$, apoCII levels were $42.0,25.0$, and $9.4 \mu \mathrm{g} / \mathrm{ml}$, respectively.

\section{ApoCII in normal and hypertriglyceridemic subjects}

Total plasma apoCII concentration in normals and in subjects with IIA, IIB, IV, and V lipoprotein phenotypes. Fig. 4 summarizes the distribution of total plasma apoCII in 32 normolipoproteinemic subjects, in 15 with type IIA, 9 with type IIB, 14 with type IV, and 5 with type $\mathrm{V}$ lipoprotein phenotypes. The mean \pm SE total plasma apoCII in 32 normolipoproteinemic subjects $(49.7 \pm 4.0 \mu \mathrm{g} / \mathrm{ml})$ was not significantly different from that in 15 subjects with IIA lipoprotein patterns $(56.2 \pm 5.4 \mu \mathrm{g} / \mathrm{ml})$. Mean \pm SE apoCII 


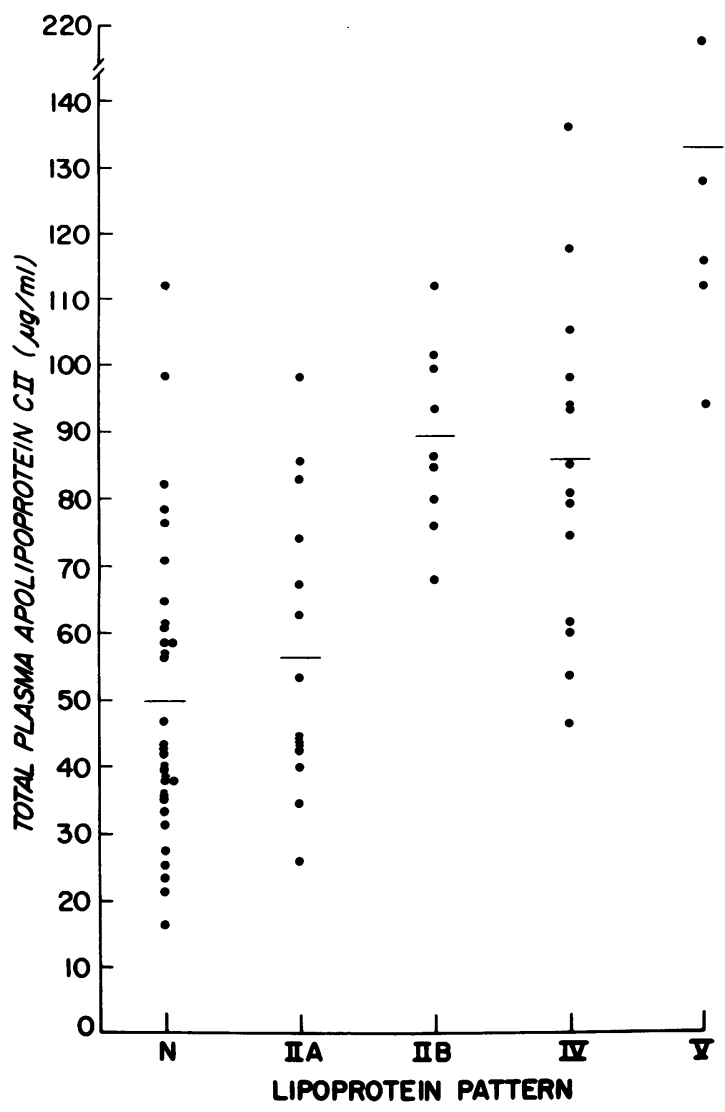

FIGURE 4 Total plasma apoCII distribution in normal and type IIA, IIB, IV, and V subjects.

in these 47 normotriglyceridemic subjects was $51.8 \pm 3.2$ $\mu \mathrm{g} / \mathrm{ml}$.

In the 9 subjects with IIB and 14 with type IV lipoprotein patterns, mean $( \pm \mathrm{SE})$ plasma apoCII concentrations did not significantly differ $(89.1 \pm 4.6$ and $85.4 \pm 6.9 \mu \mathrm{g} / \mathrm{ml}$, respectively), but were higher ( $P$ $<0.001$ ) than in normotriglyceridemic individuals.

The highest apoCII levels were quantitated in five subjects with type $\mathrm{V}$ lipoprotein patterns, $132.8 \pm 21.0$ $\mu \mathrm{g} / \mathrm{ml}$, significantly higher than those with types IIB, and IV patterns $(P<0.02)$, or normotriglyceridemic subjects $(P<0.001)$.

The mean levels of plasma apoCII in normotriglyceridemic males and females were $55.4 \pm 4.2$ and $43.9 \pm 3.9 \mu \mathrm{g} / \mathrm{ml}$, with plasma triglycerides $89.7 \pm 8.5$ and $64.5 \pm 7.7 \mathrm{mg} / \mathrm{dl}$, respectively. By analysis of covariance taking into account an ancillary variable (total plasma triglycerides) there was no significant male-female difference in total plasma apoCII.

Relationship of total plasma apoCII to plasma triglyceride. The (log) total plasma triglycerides positively correlated with total plasma apoCII in the 47 normotriglyceridemic subjects $(r=0.36 ; P<0.05)$, and in 28 hypertriglyceridemic subjects $(r=0.58, P<0.01)$,
Fig. 5. For all 75 subject the (log) triglyceride: apoCII correlation was $0.75, P<0.01$, Fig. 5 .

Relationship between plasma apoCII and HDL cholesterol. Total plasma apoCII did not correlate significantly with plasma HDL cholesterol $(n=28$; $r=-0.23, P>0.1$ ).

Distribution of apoCII in VLDL and VLDL-free plasma. The percentage of plasma apoCII in VLDLfree plasma ( $d>1.006$ fraction) in normotriglyceridemics and hypertriglyceridemics was 53.0 \pm 4.1 and $21.3 \pm 3.8 \%$, respectively $(P<0.001)$. There was a significant inverse relationship between (log) total plasma triglycerides and the percent of total plasma apoCII in VLDL-free plasma fraction in 35 subjects $(r=-0.75$, $P<0.01$ ), Fig. 6 .

Relationship between apoCII concentration and activator activity in VLDL and VLDL-free plasma in normal subjects. In 18 normotriglyceridemic subjects (arbitrarily defined by total plasma triglyceride concentration of $\leq 170 \mathrm{mg} / \mathrm{dl}$ ), the in vitro LPL activator property of the VLDL subfraction correlated positively with the VLDL apoCII concentration $(r=0.89 ; P$ $=<0.01$ ) Fig. 7. As depicted by Fig. 8 , for 25 normotriglyceridemic subjects, there was a significant positive correlation between apoCII in the plasma fraction $(d>1.006)$ and the activator concentration $(r$ $=0.65, P<0.01)$. For the 18 normals, there was also a

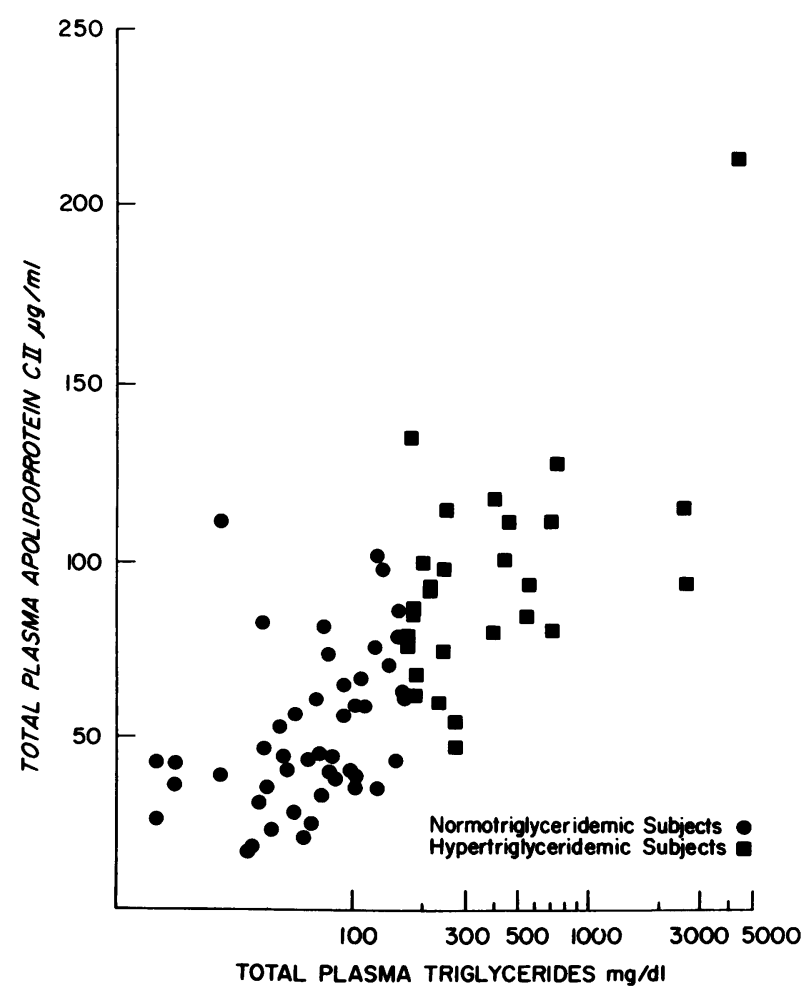

FIGURE 5 Correlation between total plasma triglycerides and apoCII in hypertriglyceridemic subjects. 


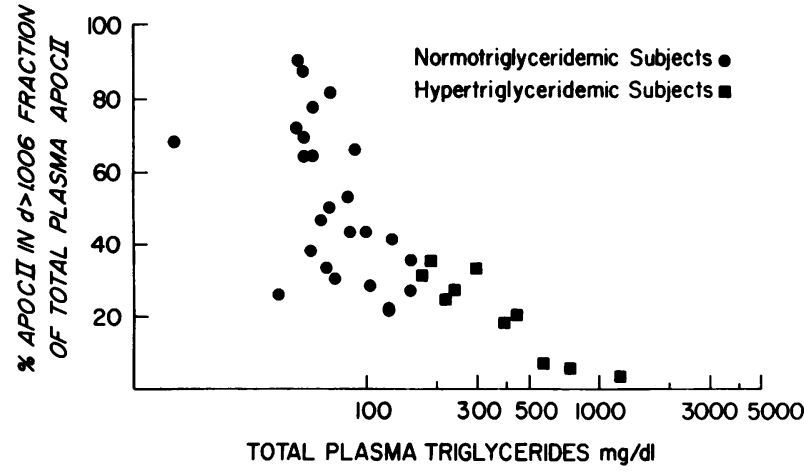

FIGURE 6 Correlation between total plasma triglyceride and percent of total plasma apoCII in VLDL-free plasma ( $d$ $>1.006)$ in normotriglyceridemic and hypertriglyceridemic subjects.

positive correlation between VLDL apoCII and VLDL protein concentrations $(r=0.94 ; P<0.01)$.

Content of apoCII and activator in VLDL protein in normotriglyceridemic and hypertriglyceridemic subjects. VLDL was isolated by ultracentrifuging plasma from 18 normal and 8 hypertriglyceridemic subjects, and the amount of VLDL protein, amount of VLDL apoCII, and percentage of VLDL protein as apoCII determined. VLDL protein from normotriglyceridemic subjects contained $9.9 \pm 0.7 \%$ apoCII, significantly more than in hypertriglyceridemic subjects, $7.1 \pm 0.9 \%(P<0.05)$.

Hypertriglyceridemic subjects had much less LPL activator concentration per milligram of VLDL protein $(73.5 \pm 10.1 \mathrm{U} / \mathrm{mg})$, then did normotriglyceridemics $(145.6 \pm 11.3 \mathrm{U} / \mathrm{mg})(P<0.01)$. From measurements of VLDL apoCII concentration and VLDL LPL activator levels, LPL activator potency per microgram of apoCII was calculated. The eight hypertriglyceridemic subjects had notably less LPL activation per microgram of VLDL apoCII than the normals, 1.02 \pm 0.04 and $1.54 \pm 0.13 \mathrm{U} / \mu \mathrm{g}$, respectively $(P<0.025)$. As the percent of total plasma apoCII in VLDL rose, the amount of LPL activator activity per microgram of VLDL apoCII fell ( $r=-0.71, P<0.01)$ (Fig. 9).

\section{DISCUSSION}

Further understanding of the role of apolipoproteins in normal and abnormal lipid transport will require specific, accurate, precise, and sensitive methods for their measurement. Methods for measurement of apoAI $(30,37,38)$, apoAII $(39)$, and apoB (40-42) that satisfy these requirements have been described.

The radioimmunoassay method. Our radioimmunoassay for the measurement of apoCII is specific. There was no appreciable cross-reactivity with apoAI, apoAII, LDL, apoCI, and apoCIII. ApoCII was not detected in lipoprotein-free plasma, $d>1.25$. Lipoprotein fractions VLDL, ILDL, $\mathrm{HDL}_{2}$, and $\mathrm{HDL}_{3}$

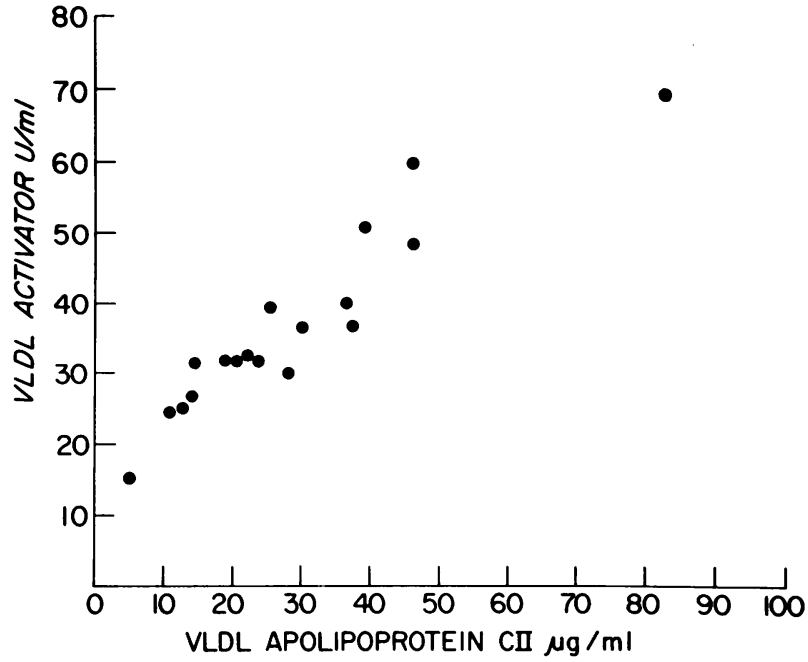

FIgURE 7 Correlation between VLDL apoCII and VLDL activator concentration in 18 normotriglyceridemic subjects.

which contain apoCII, produced parallel displacement of labeled apoCII. LDL contains approximately onethird of total plasma apoE $(\cong>10 \mathrm{mg} / \mathrm{dl})$ (43). We did not detect significant cross-reactivity with LDL nor a parallel displacement of the tracer by LDL to the standard curve. Thus we infer that the apoCII antibody did not significantly cross-react with apoE.

The method was also accurate. Plasma total apoCII was not increased by pretreatment with organic solvent to extract lipid (30). Preincubation of plasma with heating (37), did not change apoCII concentration. Delipidation with the organic solvent TMU which solubilizes apolipoproteins except apoB $(10,17)$ did not increase apoCII levels. The $10-13 \%$ content of apoCII

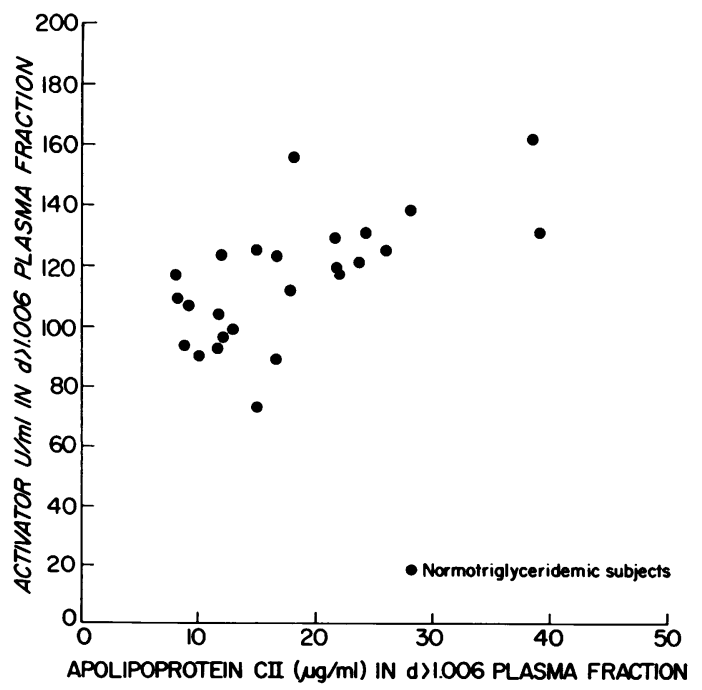

FIGURE 8 Correlation between apoCII in VLDL-free plasma $(d>1.006)$ and activator concentration in 25 normotriglyceridemic individuals. 


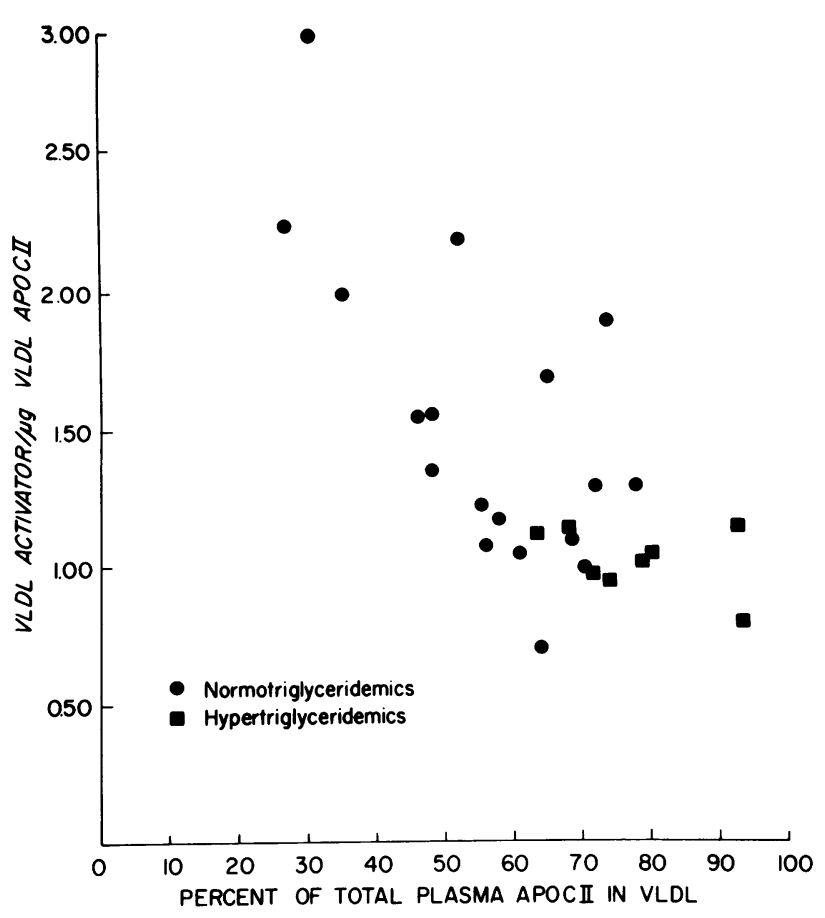

Figure 9 Correlation between percent of total plasma apoCII in VLDL and potency per microgram of immunoreactive VLDL apoCII in normotriglyceridemic and hypertriglyceridemic subjects.

in normal VLDL protein (by radioimmunoassay) reported by Miller and Schonfeld (14) is in accord withour observations. Using densitometric scanning of TMU-soluble VLDL apolipoproteins separated electrophoretically in polyacrylamide gel, Kane et al. (17) reported apoCII values ranging between 4.5 and $12.0 \%$. In our study the range of apoCII content in normal VLDL $(5.1-16.9 \%)$ is somewhat higher than that reported by Kane et al. The mean level of plasma apoCII by our radioimmunoassay ( $\cong 50 \mu \mathrm{g} / \mathrm{ml}$ in normals) is also comparable to the $40 \mu \mathrm{g} / \mathrm{ml}$ found by McConathy and Alaupovic using electroimmunoassay (18). The concentrations of normal VLDL and HDL protein are estimated to be $\cong 25$ and $150-200 \mathrm{mg} / \mathrm{dl}$, respectively $(17,30,38)$. Since $10 \%$ of VLDL protein and $1-2 \%$ of HDL protein is apoCII (14) the calculated total concentration of apoCII would be expected to be $\cong 4.0-$ $6.5 \mathrm{mg} / \mathrm{dl}$. This approximation of total plasma apoCII concentration from published work is in accord with our measurements.

The radioimmunoassay method was very sensitive, allowing quantitation of as little as $0.6 \mathrm{ng}$ of apoCII. The method was also precise, with inter- and intraday coefficients of variation being 4.9 and $3.5 \%$, respectively. The accuracy of the radioimmunoassay method is indirectly validated by the close correlation of VLDL apoCII and the CII mediated LPL activator concentration by the bioassay system.
ApoCII in normal and hypertriglyceridemic subjects. In normal and hypertriglyceridemic subjects fasting plasma triglycerides correlate positively with plasma apoCII. There is an inverse correlation between plasma triglyceride and the apoCII content of VLDL-free plasma (Fig. 6) which suggests a transfer of apoCII to VLDL from HDL as triglycerides increase.

Although total net LPL activator property in plasma does not change after acute fat ingetion, both $\mathrm{C}$ and apolipoproteins and LPL activator property are transferred from HDL to chlomicrons (10). HDL probably functions as carrier and reservoir of the $\mathrm{C}$ peptides for the metabolism of chylomicrons formed aftei triglyceride ingestion. In subjects with plasma triglyceride $\leq 170 \mathrm{mg} / \mathrm{dl}$, an average of $47 \%$ of the total apoCII is present in VLDL (Fig. 6, calculated as [total apoCII]-[apoCII in $d>1.006$ fraction]). In 10 subjects whose plasma triglycerides ranged from 171 to 1,220 $\mathrm{mg} / \mathrm{dl}$, the fraction of the total apoCII in VLDL increased to $78.7 \%$. The percent of apoCII in the $d$ $>1.006$ fraction fell as triglyceride levels increased in hyperlipemic subjects (Fig. 6). In three subjects with triglycerides to 580,768 , and $1,220 \mathrm{mg} / \mathrm{dl}$ and chylomicronemia, the percent of total apoCII in VLDLfree plasma was $7.0,6.3$, and $4 \%$, respectively.

As plasma triglycerides and total apoCII increase in parallel the distribution of apoCII shifts, with progressive reduction of apoCII in the VLDL-free plasma fraction.

The VLDL-free plasma fraction includes apoCIIcontaining lipoporteins, ILDL, and HDL. The former is considered to be an intermediate product of VLDL and chylomicron catabolism and contains some apoCII. These considerations suggest that as triglyceride levels increase, HDL is progressively depleted of apoCII. This would lead to limited reserves in HDL for transfer to apoCII to VLDL to facilitate catabolism of newly synthesized VLDL and chylomicron triglyceride. The apoCII content per milligram of VLDL protein is much lower in hypertriglyceridemic patients, possibly because of insufficient transfer from HDL.

In hypertriglyceridemia, reductions of immunoreactive apoCII in VLDL are accompanied by decreased LPL activation per milligram of total VLDL protein. Hypertriglyceridemic subjects have less LPL activator per unit of VLDL apoCII. As total plasma triglycerides and the proportion of total apoCII in VLDL increase, the LPL activator potency per unit of VLDL apoCII progressively falls (Fig. 9). We speculate that VLDL apoCII in hypertriglyceridemic subjects may be inhibited in its activating property on LPL. ApoCI, apoAI, apoE, apoCIII are inhibitors of human adipose tissue LPL (4). These peptides, possibly along with the apoE, the arginine-rich peptide, are also transferred from HDL to VLDL during alimentary 
lipemia, and an increase in their concentration relative to apoCII in hypertriglyceridemic VLDL is suggested by our data. It is possible that the addition of very high concentrations of VLDL triglyceride to the triolein substrate might somewhat dilute labeled triolein, leading to an apparent reduction in LPL in the more hypertriglyceridemic subjects. Although this possibility cannot be completely ruled out, it is unlikely, as serial dilutions of a lipemic sample gave correspondingly lower concentrations of activator. With fixed amounts of enzyme and the usual amount of labeled and unlabeled triolein the addition of triolein (within the range of triglyceride mass added in an unknown sample) did not decrease its activator concentration. Also the unlabeled substrate concentration is in excess of the amount of VLDL triglyceride added and the assay operates under conditions of substrate excess.

An inverse correlation between increasing plasma triglycerides and plasma post-heparin extrahepatic LPL has been noted, using selective immunochemical methods (44). In patients with hypertriglyceridemia, however, plasma post-heparin lipolytic activity is normal, or low normal (44). Disorders involving an absolute or relative deficiency of apoCII might then lead to hypertriglyceridemia, as would reductions of extrahepatic lipoprotein lipase as in type I hyperlipoproteinemia. The recent report of a severely hypertriglyceridemic patient with absent apoCII (13) illustrates the importance of apoCII and LPL in triglyceride homeostasis. The deficiency of apoCII in VLDL in some of the hypertriglyceridemic subjects of this study suggests another apoCII-hypertriglyceridemia interaction. Further research as suggested in the current studies is obviously necessary to understand the fundamental nature of these abnormalities.

\section{ACKNOWLEDGMENTS}

We thank Doctors O. D. Taunton and A. M. Gotto of Lipid Research Clinic, Baylor College of Medicine, Houston, Texas, for suppling us with pure A apolipoproteins; Ms. Paula M. Steiner and the staff of the Lipid Research Clinic Core Laboratory for their assistance in quantification of lipid and lipoportein lipids; Ms. Barbara A. Hynd, Mr. Kent Robinson, and Mr. David Brady for technical assistance; and Ms. Alta S. Ressler for typing this manuscript.

This work was supported by grants from the Lipid Research Clinic Program of the National Heart, Lung and Blood Institute (NIH NHLI 72-2914-L) and the General Clinical Research Centers Program of the Division of Research Resources, NIH (RR00068-15).

\section{REFERENCES}

1. Kashyap, M. L., L. S. Srivastava, C. Y. Chen, G. Perisutti, M. Campbell, R. F. Lutmer, and C. J. Glueck. 1976. Radioimmunoassay of human plasma apolipoprotein CII. Clin. Res. 24: 528A. (Abstr.)

2. Havel, R. J., V. G. Shore, B. Shore, and D. M. Bier. 1970. Role of specific glycopeptides of human serum lipoproteins in the activation of lipoprotein lipase. Circ. Res. 27: 595-600.

3. LaRosa, J. C., R. I. Levy, P. Herbert, S. E. Lux, and D. S. Fredrickson. 1970. A specific apoprotein activator for lipoprotein lipase. Biochem. Biophys. Res. Commun. 41: 57-62.

4. Ekman, R., and P. Nilsson-Ehle. 1975. Effects of apolipoproteins on lipoprotein lipase activity of human adipose tissue. Clin. Chim. Acta. 63: 29-35.

5. Twu, J-S., A. S. Garfinkel, and M. C. Schotz. 1976. Purification and characterization of lipoprotein lipase from human heart. Atherosclerosis. 24: 119-128.

6. Havel, R. J., C. J. Fielding, T. Olivecrona, V. G. Shore, P. E. Fielding, and T. Egelrud. 1973. Cofactor activity of protein components of human very low density lipoproteins in the hydrolysis of triglycerides by lipoprotein lipase from different sources. Biochemistry. 12: 18281833.

7. Krauss, R. M., P. N. Herbert, R. I. Levy, and D. S. Fredrickson. 1973. Further observations of the activation and inhibition of lipoprotein lipase by apolipoproteins. Circ. Res. 33: 403-411.

8. Östlund-Lindquist, A-M., and P-H. Iverius. 1975. Activation of high purified lipoprotein lipase from bovine milk. Biochem. Biophys. Res. Commun. 65: 1447-1455.

9. Ganesan, D., R. H. Bradford, G. Ganesan, W. J. McConathy, P. Alaupovic, and H. B. Bass. 1975. Purified postheparin plasma lipoprotein lipase in primary hyperlipoproteinemias. J. Appl. Physiol. 39: 1022-1033.

10. Havel, R. J., J. P. Kane, and M. L. Kashyap. 1973. Interchange of apolipoproteins between chylomicrons and high density lipoproteins during alimentary lipemia in man. J. Clin. Invest. 52: 32-38.

11. Eisenberg, S., and R. I. Levy. 1975. Lipoprotein metabolism. Adv. Lipid Res. 13: 1-89.

12. Quarfordt, S. H., A. Frank, D. M. Shames, M. Berman, and D. Steinberg. 1970. Very low density lipoprotein triglyceride transport in type IV hyperlipoproteinemia and the effects of carbohydrate-rich diets. J. Clin. Invest. 49: 2281-2297.

13. Breckenridge, C. W., J. A. Little, G. Steiner, A. Chow, and M. Poapst. 1976. Hyperlipoproteinemia associated with an absence of CII apoprotein in plasma lipoproteins. Circulation. 54 (Suppl. II): 25. (Abstr.)

14. Miller, J., and G. Schonfeld. 1975. Radioimmunoassay for the apolipoprotein CII of human very low density lipoproteins. Circulation. 51 (Suppl. II): 274. (Abstr.)

15. Chu, P., A. L. Miller, and G. L. Mills. 1976. Assay of an activator for lipoprotein lipase. Clin. Chim. Acta. 66: 281-286.

16. Rogers, M. P., D. Barnett, and D. S. Robinson. 1976. Clearing factor lipase (lipoprotein lipase) activator. A method for the measurement of the net activating ability of human sera. Atherosclerosis. 24: 551-564.

17. Kane, J. P., T. Sata, R. L. Hamilton, and R. J. Havel. 1975. Apoprotein composition of very low density lipoproteins of human serum. J. Clin. Invest. 56: 1622-1634.

18. McConathy, W. J., and P. Alaupovic. 1976. Proceedings of the International Symposium of Lipoprotein Structure and Metabolism. In press.

19. Havel, R. J., H. A. Eder, and J. H. Bragdon. 1955. The distribution and chemical composition of ultracentrifugally separated lipoproteins in human serum. J. Clin. Invest. 34: 1345-1353.

20. Brown, W. V., R. I. Levy, and D. S. Frederickson. 1969. Studies of the proteins in human plasma very low density lipoproteins. J. Biol. Chem. 244: 5687-5694.

21. Brown, W. V., R. I. Levy, and D. S. Fredrickson. 1970. 
Further characterization of apolipoproteins from human plasma very low density lipoproteins. J. Biol. Chem. 245: 6588-6594.

22. Weber, K., and M. Osborn. 1969. The reliability of molecular weight determinations by dodecyl sulfatepolyacrylamide gel electrophorsis. J. Biol. Chem. 244: 4406-4412.

23. Newbould, B. B. 1965. Production of allergic encephalomyelitis in rats by injections of spinal cord adjuvant into the inguinal lymph nodes. Immunology. 9: 613-614.

24. Vaitukaitis, J., J. B. Robbins, E. Nieschlag, and G. T. Ross. 1971. A method for producing specific antisera with small doses of immunogen. J. Clin. Endocrinol. 33: 988-991.

25. Greenwood, F. C., W. M. Hunter, and J. S. Glover. 1963. The preparation of ${ }^{131}$ I-labelled human growth hormone of high specific radioactivity. Biochem. J. 89: 114-123.

26. Schotz, M. C., A. S. Garfinkel, R. J. Huebotter, and J. E. Stewart. 1970. A rapid assay for lipoprotein lipase. J. Lipid Res. 11: 68-69.

27. Bier, D. M., and R. J. Havel. 1970. Activation of lipoprotein lipase by lipoprotein fractions of human serum. J. Lipid Res. 11: 565-570.

28. Lowry, O. H., N. J. Rosebrough, A. L. Farr, and R. J. Randall. 1951. Protein measurement with the Folin phenol reagent. J. Biol. Chem. 193: 265-275.

29. Lipid Research Clinics Program. 1974. National Institutes of Health, Department of Health, Education and Welfare Publication No. (NIH) 75-628: 9-50.

30. Schonfeld, G., and B. Pfleger. 1974. The structure of human high density lipoprotein and the levels of apolipoprotein A-I in plasma as determined by radioimmunoassay. J. Clin. Invest. 54: 236-246.

31. Kane, J. P. 1973. A rapid electrophoretic technique for identification of subunit species of apoproteins in serum lipoproteins. Anal. Biochem. 53: 350-364.

32. Lipid Research Clinics Program. 1974. National Institutes of Health, Department of Health, Education and Welfare Publication No. (NIH) 75-628: 74-81.

33. Snedecor, G. R., and W. G. Cochran. 1967. In Statistical Methods. Iowa State University Press, Ames. 6th edition. 135-198.

34. Jackson, R. L., and A. M. Gotto. 1972. A study of the cystine-containing apolipoprotein of human plasma high density lipoproteins: characterization of cyanogen bromide and tryptic fragments. Biochim. Biophys. Acta. 285: 36-47.

35. Brown, W. V., and M. L. Baginsky. 1972. Inhibition of lipoprotein lipase by an apoprotein of human very low density lipoprotein. Biochem. Biophys. Res. Commun. 46: 375-382.

36. Brewer, H. B., Jr., R. Schulman, P. Herbert, R. Ronan, and $K$. Wehrly. 1974. The complete amino acid sequence of alanine apolipoprotein (apo C-III), an apolipoprotein from human plasma very low density lipoproteins. J. Biol. Chem. 249: 4975-4984.

37. Karlin, J. B., D. J. Juhn, J. I. Starr, A. M. Scanu, and A. H. Rubenstein. 1976. Measurement of human high density lipoprotein apolipoprotein $\mathrm{AI}$ in serum by radioimmunoassay. J. Lipid Res. 17: 30-37.

38. Albers, J. J., P. W. Wahl, V. G. Cabana, W. R. Hazzard, and J. J. Hoover. 1976. Quantitation of apolipoprotein A-I of human plasma high density lipoprotein. Metab. (Clin. Exp.). 25: 633-644.

39. Mao, S. J. T., A. M. Gotto, Jr., and R. L. Jackson. 1975. Immunochemistry of human plasma high density lipoproteins. Radioimmunoassay of apolipoprotein A-II. Biochemistry. 14: 4127-4131.

40. Schonfeld, G., R. S. Lees, P. K. George, and B. Pfleger. 1974. Assay of total plasma apolipoprotein B concentration in human subjects. J. Clin. Invest. 53: 1458-1467.

41. Bautovich, G. J., L. A. Simons, P. F. Williams, and J. R. Turtle. 1975. Radioimmunoassay of human plasma apolipoproteins. Part 1. Assay of apolipoprotein-B. Atherosclerosis. 21: 217-234.

42. Alberts, J. J., V. G. Cabana, and W. R. Hazzard. 1975. Immunoassay of human plasma apolipoprotein B. Metab. (Clin. Exp.). 24: 1339-1351.

43. Curry, M. D., W. J. McConathy, P. Alaupovic, J. H. Ledford, and M. Popović. 1976. Determination of human apolipoprotein $\mathrm{E}$ by electroimmunoassay. Biochim. Biophys. Acta. 439: 413-425.

44. Huttunen, J. K., C. Ehnholm, M. Kekki, and E. A. Nikkila. 1976. Post-heparing plasma lipoprotein lipase and hepatic lipase in normal subjects and in patients with hypertriglyceridemia: correlations to sex, age and various parameters of triglyceride metabolism. Clin. Sci. Mol. Med. 50: 249-260. 